Теорія Ймовір. та Матем. Статист. Вип. 79, 2008
Theor. Probability and Math. Statist.

No. 79, 2009, Pages 139-142

S 0094-9000(09)00775-3

Article electronically published on December 28, 2009

\title{
ON THE ABSOLUTE CONTINUITY OF FIXED POINTS OF SMOOTHING TRANSFORMS
}

UDC 519.21

\author{
SERGIY POLOTS'KIY
}

\begin{abstract}
Let the distribution of a nonnegative random variable $W$ be such that $W \stackrel{d}{=} \sum_{i=1}^{J} Y_{i} W_{i}$, where $\left\{Y_{i}: i=1, \ldots, J\right\}$ are some positive random variables. Under some moment conditions imposed on $Y_{i}$ we show that the distribution of $W$ is a mixture of the atom at the origin and an absolutely continuous component.
\end{abstract}

\section{Introduction AND STATEMENT OF the RESUlt}

Consider a family of positive random points $\left\{Y_{i}: i=1, \ldots, J\right\}$, where the number $J$ can be deterministic or random as well as finite or infinite with positive probability. Let $\mathcal{L}(\xi)$ denote the distribution of a random variable $\xi$ and let $\mathcal{P}^{+}$be the set of probability measures on $[0, \infty)$. The definitions given below can be found in the book [5]. A transform

$$
\mathbb{T}: \mathcal{P}^{+} \rightarrow \mathcal{P}^{+} \cup\left\{\delta_{\infty}\right\}, \quad \mathcal{L}(Z) \mapsto \mathcal{L}\left(\sum_{i=1}^{J} Y_{i} Z_{i}\right)
$$

is called a homogeneous smoothing transform if $\left\{Z_{i}: i \in \mathbf{N}\right\}$ are independent copies of a random variable $Z$ and if the sequence $\left\{Z_{i}: i \in \mathbf{N}\right\}$ is independent of $\left\{Y_{i}: i=1, \ldots, J\right\}$.

The distribution of a nonnegative random variable $W$ is called a fixed point of a smoothing transform if the following equality holds for the distributions:

$$
W \stackrel{d}{=} \sum_{i=1}^{J} Y_{i} W_{i}
$$

where $\left\{W_{i}\right\}$ are independent copies of $W$ and if the sequence $\left\{W_{i}\right\}$ is independent of $\left\{Y_{i}: i=1, \ldots, J\right\}$.

Let $\alpha \in(0,1]$ be given. We say that a distribution $\mu_{\alpha}$ is an $\alpha$-elementary fixed point of a transform $\mathbb{T}$ if its Laplace-Stieltjes transform $\varphi_{\alpha}$ is such that

$$
\lim _{s \rightarrow+0} \frac{1-\varphi_{\alpha}(s)}{s^{\alpha}}=m
$$

for some finite number $m>0$. Note that a fixed point is 1-elementary if and only if it has a finite mean. The set of elementary fixed points is the union of all $\alpha$-elementary fixed points with respect to $\alpha \in(0,1]$. A fixed point is called nonelementary if there is no $\alpha \in(0,1]$ for which the point is $\alpha$-elementary.

Corollary 2.4.1 of [5] implies that a 1-fixed point is either a degenerate distribution at the point 1 or it is a mixture of an atom at the origin and a purely continuous component.

2000 Mathematics Subject Classification. Primary 60J80, 60E99; Secondary 60G42.

Key words and phrases. Smoothing transform, absolute continuity, fixed points. 
Now we state the main result of the paper, which improves Corollary 2.4.1 of 5 in the sense that it contains conditions under which the purely continuous component is absolutely continuous.

Theorem 1.1. a) Assume that $J=\infty$ almost surely and that $\mu$ is a nondegenerate 1-elementary fixed point. If, for some $j \in \mathbf{N}$ and $a>0$,

$$
\mathrm{E} Y_{j}^{-a}<\infty
$$

then $\mu$ is an absolutely continuous distribution.

b) Let $\mu$ be an $\alpha$-elementary fixed point, $\alpha \in(0,1)$. Then, for some $p \in[0,1)$,

$$
\mu(d x)=p \delta_{0}(d x)+(1-p) \nu(d x)
$$

where $\nu$ is an absolutely continuous distribution.

The absolute continuity of fixed points of probability distributions is studied by other authors as well. In particular, the paper [3] contains sufficient conditions for the absolute continuity of the distribution of the limit random variable for the Bellman-Harris branching process satisfying the equality $Z \stackrel{d}{=} A\left(Z_{1}+\cdots+Z_{N}\right)$, where $Z_{1}, Z_{2}, \ldots$ are independent copies of $Z$ such that the sequence $\left\{Z_{i}\right\}$ is independent of $(A, N)$. In the papers [1] and [4, sufficient conditions are found for the existence of an absolutely continuous component of a distribution satisfying equality (11) with $J<\infty$ almost surely. Sufficient conditions are given in 2] for the absolute continuity of a fixed point for the case where the $Y_{i}$ are points of a Poisson point process.

\section{Proof of Theorem 1.1}

We study fixed points with a finite mean. Denote by $\phi(t)$ the characteristic function of such a fixed point. According to the assumption of the theorem, $J=\infty$ almost surely. Thus equality (1) is equivalent to the following one:

$$
\phi(t)=\mathrm{E} \prod_{i=1}^{\infty} \phi\left(Y_{i} t\right)
$$

We need some auxiliary results.

Lemma 2.1. If $J=\infty$ almost surely, then

$$
\lim _{t \rightarrow \infty}|\phi(t)|=0 .
$$

Proof. Put $q:=\varlimsup_{t \rightarrow \infty}|\phi(t)|$. Equality (3) implies that

$$
q \leq \varlimsup_{t \rightarrow \infty} \mathrm{E} \prod_{i=1}^{\infty}\left|\phi\left(Y_{i} t\right)\right|=\prod_{i=1}^{\infty} q .
$$

It is clear that the latter inequality may hold either for $q=0$ or for $q=1$ only. Now we prove that $q<1$, whence we conclude that $q=0$. Since a fixed point has a finite mean, we have $\mathrm{E} \sum_{i=1}^{\infty} Y_{i}=1$. Thus the expectation of the number of those $Y_{i}$ that do not exceed 1 is strictly greater than 1 . Thus one can choose $\delta \in(0,1)$ such that $\mathrm{E} T_{\delta}>1$, where $T_{\delta}:=\#\left\{i: Y_{i} \in[\delta, 1]\right\}$.

Assume that $q=1$. Corollary 2.4.1 of [5] implies that the distribution of a fixed point is continuous. Thus $|\phi(t)|<1$ for all nonzero real $t$. Fix an arbitrary $\varepsilon>0$ and choose $t_{1}$ and $t_{2}$ such that $t_{1}<\delta t_{2}$ and

$$
\left|\phi\left(t_{1}\right)\right|=\left|\phi\left(t_{2}\right)\right|=1-\varepsilon \quad \text { and } \quad|\phi(t)|<1-\varepsilon \quad \text { for } t_{1}<t<t_{2} .
$$


Further

$$
1-\varepsilon=\left|\phi\left(t_{2}\right)\right| \leq \mathrm{E} \prod_{i=1}^{\infty}\left|\phi\left(Y_{i} t_{2}\right)\right| \leq \mathrm{E} \prod_{r \in R}\left|\phi\left(Y_{r} t_{2}\right)\right|,
$$

where $R=\left\{r: t_{1} / t_{2}<Y_{r} \leq 1\right\}$. Thus we derive from the latter inequality that

$$
1-\varepsilon \leq \mathrm{E}(1-\varepsilon)^{T_{\delta}}
$$

for all sufficiently small $\varepsilon$. This result contradicts the inequality $\mathrm{E} T_{\delta}>1$.

Lemma 2.2. Let

$$
f(t) \leq p \mathrm{E} f(A t)
$$

for a nonnegative bounded Borel function $f$, positive random variable $A$, some $p \in(0,1)$, $t_{0} \geq 0$ and all $t \geq t_{0}$. If $p \mathrm{E}\left(A^{-a}\right)<1$ for some $a>0$, then

$$
f(t)=O\left(t^{-a}\right), \quad t \rightarrow \infty .
$$

The proof of Lemma 2.2 can be found in 3 .

The following result is Lemma 5.3.2 in the monograph [5].

Lemma 2.3. Let $\eta_{1}$ and $\eta_{2}$ be independent nonnegative random variables with the distributions $\chi_{1}$ and $\chi_{2}$, respectively. If $\chi_{1}$ is an absolutely continuous distribution with density $g_{1}$, then the distribution $\chi$ of the random variable $\eta_{1} \eta_{2}$ is given by $\chi(d x)=$ $p \delta_{0}(d x)+g(x) d x$, where $p:=\chi_{2}\{0\} \in[0,1)$ and $g(x):=\int_{+0}^{\infty} y^{-1} g_{1}\left(x y^{-1}\right) \chi_{2}(d y)$ is the density of the absolutely continuous component of $\chi$. If the $n$-th derivative of $g_{1}$ is bounded and continuous, then the $n$-th derivative of $g$ exists and is continuous.

Now we turn to the proof of the theorem. Choose an arbitrary fixed $\varepsilon \in(0,1)$ and $\delta>0$. Put $N_{\delta}:=\sum_{i=1}^{\infty} 1_{\left\{Y_{i}>\delta\right\}}$. Since the mean of a fixed point is finite, we have $\mathrm{E} \sum_{i=1}^{\infty} Y_{i}=1$. Thus

$$
1 \geq \mathrm{E} \sum_{i=1}^{\infty} Y_{i} 1_{\left\{Y_{i}>\delta\right\}} \geq \delta \mathrm{E} N_{\delta},
$$

whence we conclude that $N_{\delta}<\infty$ almost surely. By the assumption of the theorem, $N_{\delta} \uparrow \infty$ as $\delta \downarrow 0$. Lemma 2.1 implies that there exists $t_{\varepsilon}>0$ such that $|\phi(t)|<\varepsilon$ for all $t>t_{\varepsilon}$. Thus we obtain from equality (3) that

$$
|\phi(t)| \leq \mathrm{E} \prod_{i=1}^{\infty}\left|\phi\left(Y_{i} t\right)\right| \leq \mathrm{E}\left|\phi\left(Y_{j} t\right)\right|\left[\varepsilon^{N_{\delta}-1} 1_{\left\{N_{\delta} \geq 1\right\}}+1_{\left\{N_{\delta}=0\right\}}\right]=p_{\varepsilon, \delta} \mathrm{E}\left|\phi\left(\widetilde{Y}_{j} t\right)\right|
$$

for $t>t_{\varepsilon} / \delta$, where $p_{\varepsilon, \delta}:=\mathrm{E} \varepsilon^{N_{\delta}-1} 1_{\left\{N_{\delta} \geq 1\right\}}+\mathrm{P}\left\{N_{\delta}=0\right\}, Y_{j}$ is a random variable whose distribution is as in Theorem 1.1, and $\widetilde{Y}_{j}$ is a positive random variable whose distribution is determined by

$$
\mathrm{E} g\left(\widetilde{Y}_{j}\right)=\frac{1}{p_{\varepsilon, \delta}} \mathrm{E} g\left(Y_{j}\right)\left[\varepsilon^{N_{\delta}-1} 1_{\left\{N_{\delta} \geq 1\right\}}+1_{\left\{N_{\delta}=0\right\}}\right]
$$

considered for an arbitrary nonnegative bounded Borel function $g$. According to the Lebesgue bounded convergence theorem, $p_{\varepsilon, \delta} \rightarrow 0$ as $\delta \downarrow 0$ and

$$
p_{\varepsilon, \delta} \mathrm{E} \tilde{Y}_{1}^{-a}=\mathrm{E} Y_{1}^{-a}\left[\varepsilon^{N_{\delta}-1} 1_{\left\{N_{\delta} \geq 1\right\}}+1_{\left\{N_{\delta}=0\right\}}\right] \rightarrow 0 .
$$

Thus $p_{\varepsilon, \delta}<1$ for sufficiently small $\delta>0$ and $p_{\varepsilon, \delta} \mathrm{E}\left(\tilde{Y}_{1}\right)^{-a}<1$. Hence

$$
|\phi(t)|=O\left(|t|^{-a}\right), \quad|t| \rightarrow \infty,
$$

in view of Lemma 2.2 and since the function $|\phi(t)|$ is even. If $a>1$, then $|\phi(t)|$ is integrable on the whole axis. Thus a fixed point is absolutely continuous. Assume that $a \in(0,1]$. Choose a natural number $n$ such that $n a>1$. For all (nonrandom) nonnegative 
$\alpha_{1}, \ldots, \alpha_{n}$, not all of which are equal to 1 , the distribution of $\alpha_{1} W_{1}+\cdots+\alpha_{n} W_{n}$ is absolutely continuous, since relation (4) implies that

$$
\left|\mathrm{E} \exp \left(i t\left(\alpha_{1} W_{1}+\cdots+\alpha_{n} W_{n}\right)\right)\right|=O\left(|t|^{-n a}\right), \quad|t| \rightarrow \infty .
$$

Therefore

$$
\mathrm{P}\left\{\sum_{i=1}^{n} Y_{i} W_{i} \in C \mid \sigma\left(Y_{j}: j \in \mathbf{N}\right)\right\}=0 \quad \text { almost surely }
$$

for an arbitrary Borel set $C \subset[0, \infty)$ of zero Lebesgue measure.

Thus

$$
\mathrm{P}\left\{\sum_{i=1}^{n} Y_{i} W_{i}+\sum_{i=n+1}^{\infty} Y_{i} W_{i} \in C \mid \sigma\left(Y_{j}: j \in \mathbf{N}\right)\right\}=0
$$

almost surely. Passing to the mathematical expectation in the latter equality, we get

$$
\mathrm{P}\left\{\sum_{i=1}^{\infty} Y_{i} W_{i} \in C\right\}=0,
$$

whence we obtain the absolute continuity of fixed points with finite mean.

Now we study $\alpha$-elementary fixed points, $\alpha \in(0,1)$. Consider the following modified transform:

$$
\mathbb{T}_{\alpha}: \mathcal{P}^{+} \rightarrow \mathcal{P}^{+} \cup\left\{\delta_{\infty}\right\}, \quad \mathcal{L}(Z) \mapsto \mathcal{L}\left(\sum_{i=1}^{J} Y_{i}^{\alpha} Z_{i}\right) .
$$

By Proposition 3.4.1 of [5], the distribution $\mu_{\alpha}$ of an $\alpha$-elementary fixed point is such that

$$
\mu_{\alpha}(x, \infty)=\int_{0}^{\infty} s_{\alpha}\left(x t^{-1 / \alpha}, \infty\right) \mu_{1}(d x), \quad x>0,
$$

where $s_{\alpha}$ is a strictly stable positive distribution with index $\alpha$ and where $\mu_{1}$ is a fixed point with the finite mean of the modified transform $\mathbb{T}_{\alpha}$. By Lemma 2.3, the distribution $\mu_{\alpha}$ admits representation (2), since all stable distributions are absolutely continuous.

\section{ACKNOWLEDGEMENT}

The author is grateful to his scientific advisor Olexander M. Iksanov for the careful reading of the paper and many useful comments.

\section{BIBLIOGRAPHY}

1. J. D. Biggins and D. R. Grey, Continuity of limit random variables in the branching random walk, J. Appl. Prob. 16 (1979), 740-749. MR.549554 (80j:60107)

2. A. Iksanov and Z. Jurek, On fixed points of Poisson shot noise transforms, Adv. Appl. Prob. 34 (2002), 798-825. MR1938943(2003i:60021)

3. Q. Liu, Asymptotic properties of supercritical age-dependent branching processes and homogeneous branching random walks, Stoch. Proc. Appl. 82 (1999), 61-87. MR.1695070|(2001b:60103)

4. Q. Liu, Asymptotic properties and absolute continuity of laws stable by random weighted mean, Stoch. Proc. Appl. 95 (2001), 83-107. MR1847093 (2002e:60141)

5. O. M. Iksanov, Random Series of a Special Form, Branching Random Walks, and SelfDecomposability, Zirka, Kyiv, 2007. (Ukrainian)

Faculty for Cybernetics, National Taras Shevchenko University, Academician Glushkov Avenue 6, Kyiv 03127, Ukraine

E-mail address: pilot_ser@mail.ru

Received 27/FEB/2008 